Management options

\title{
Radiotherapy and chemotherapy for inoperable non-small cell lung cancer
}

\author{
Carol Bleasdale, Bleddyn Jones
}

\begin{abstract}
Summary
Non-small cell lung cancer is a major cause of mortality and significant morbidity in the UK. The majority of patients are inoperable and the optimum management of these patients requires a multidisciplinary approach involving the cooperation of respiratory physicians, thoracic surgeons and clinical oncologists (radiotherapists). Treatment techniques are constantly being refined and new approaches developed.
\end{abstract}

Keywords: non-small cell lung cancer, radiotherapy, management, chemotherapy

\begin{tabular}{|l|}
\hline Management options \\
\hline - radiotherapy: $\quad \begin{array}{l}\text { radical } \\
\text { palliative } \\
\text { - chemotherapy: }\end{array}$ \\
single agent \\
- surveillance $\quad$ multi-agent \\
\hline
\end{tabular}

Box 1

\begin{tabular}{|l|}
\hline Case history 1 \\
\hline - age: 67 years \\
past medical history: aortic \\
aneurysm repair 1972; angina \\
- December 1989: myocardial \\
infarction; routine chest X-ray: left \\
upper lobe mass; asymptomatic \\
- April 1990: bronchoscopy normal; \\
fine needle aspiration of mass; \\
histology: adenocarcinoma; T2 N0 \\
M0 \\
- May 1990: radical radiotherapy to \\
upper left lobe and adjacent \\
mediastinum (dose: 55 Gy in 20 \\
fractions over 28 days) \\
December $1990:$ coronary artery \\
bypass graft performed \\
- June 1994: patient well, occasional \\
angina; no respiratory symptoms \\
\hline
\end{tabular}

Box 2

Clatterbridge Centre for Oncology, Bebington, Wirral, Merseyside L63 4JY, UK

C Bleasdale

B Jones

Accepted 20 February 1995
Less than $20 \%$ of all new cases of non-small cell lung cancer are operable ${ }^{1}$ and therefore the majority of patients have inoperable cancer at presentation. It is important that all clinicians dealing with lung cancer patients are aware of the management options for inoperable cases (see box 1). In addition, some new developments are considered.

\section{Radiotherapy}

Radiotherapy is the treatment of cancers by ionising radiation, which causes damage to the DNA of living cells. This damage is caused by reactive oxygen species and free radicals generated by the action of ionising radiation on intracellular molecules. The DNA damage may cause cell death which usually occurs during attempted cell division (mitosis). It is known that squamous cancer cells will divide and proliferate at relatively short intervals of two to five days (the potential doubling time). This means that prolonged radiotherapy treatment times (over six weeks) may allow tumour cells to continue to proliferate and repopulate during the treatment. This is a potential cause of treatment failure and has led to the introduction of shortened or accelerated treatment regimes. The dose-limiting factor in radiotherapy is the maximum dose that the surrounding normal tissues can tolerate and still maintain normal function. In order to give large doses of radiotherapy, it is necessary to give the treatment in a series of small doses or fractions, over a period of time.

\section{RADICAL RADIOTHERAPY}

This is given with curative intent, the indications being similar to those for surgery, but also including those tumours in which surgery is not technically feasible (eg, when the tumour is adherent to major blood vessels) as well as tracheal tumours and those patients who are medically unsuitable or who decline surgery, but would otherwise be operable. This is therefore a relatively small group (approximately $15 \%$ of all new cases) but it is important since cure is possible. ${ }^{1-3}$

Treatment is usually protracted and complex. Computed tomography (CT) scans of the thorax are required to outline the tumour volume as well as to delineate clinically relevant normal structures including the contralateral lung and the spinal cord, which must receive a considerably lower dose than the tumour for preservation of function. Computer-generated dosimetry plans are produced to ascertain the doses to the tumour and the normal tissues of clinical significance. Techniques usually involve multiple radiotherapy fields from different directions in order to achieve an optimum dose distribution (figure 1).

The treatment courses usually last between three and six weeks to give a total dose of 40-60 Gy to the tumour volume, and treatment fractions are given daily on weekdays (ie, five days per week). One- and two-year survival rates after radical radiotherapy are $30-40 \%$ and $10-15 \%$, respectively. ${ }^{4,5}$ Many patients continue to die with uncontrolled local disease $(40-60 \%)$ and with metastatic disease $(70-90 \%) .^{5}$

Out-patient treatment is often provided since acute side effects are not usually disabling. These usually occur later in the treatment course and include acute odynophagia and dysphagia (due to oesophageal inflammation), mild lethargy and fatigue. These side effects are usually relieved by suitable medication (eg, mucaine for dysphagia) and appropriate dietary modifications. Nausea, vomiting, and skin reactions are not features of radiotherapy to the thorax. The patient's general practitioner may need to reassure the patient that these side effects are self limiting, and may need to prescribe medication such as antacids to relieve the oesophagitis.

Late side effects (which occur 6-12 months after the radiotherapy) include pneumonitis and pulmonary fibrosis in the high-dose treatment volume, which may cause cough and impairment of pulmonary function resulting in dyspnoea. 


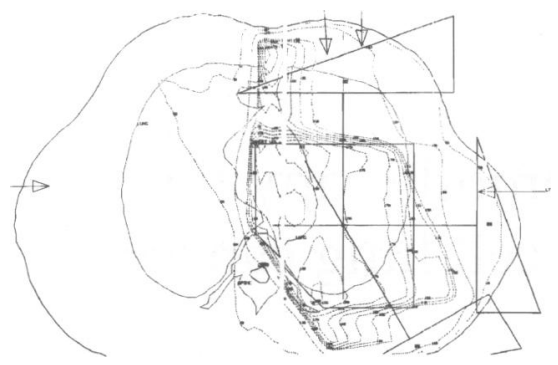

Figure 1 Dose distribution obtained by computer planning for radical treatment of lung cancer

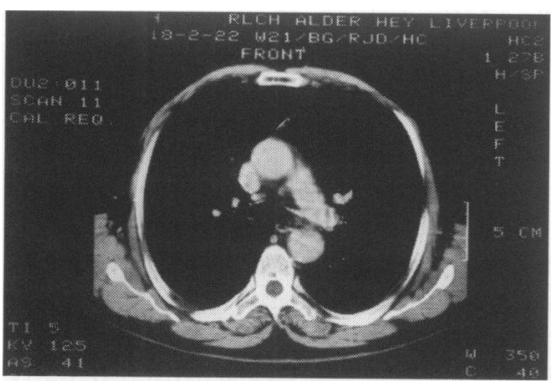

Figure 2 CT scan of the thorax of case 1, showing a left hilar mass invading the mediastinum

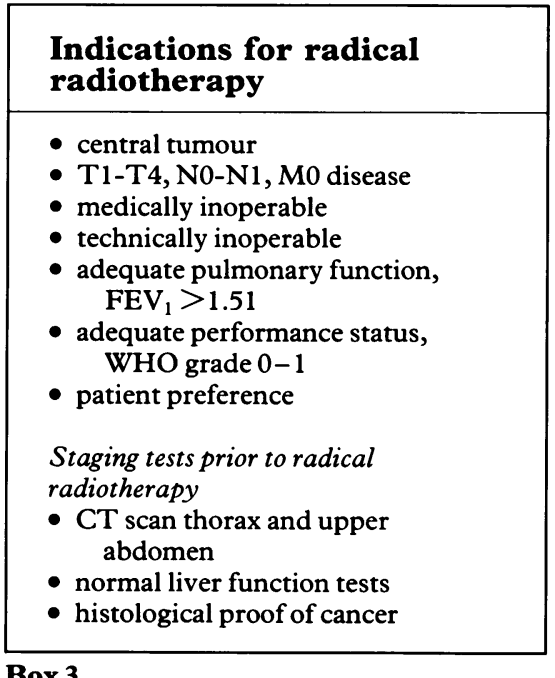

\begin{tabular}{|l|}
\hline Indications for palliative \\
treatment of primary \\
tumours \\
\hline - advanced disease \\
- poor performance status or \\
pulmonary function \\
- intercurrent medical illness \\
pain alleviate cough haemoptysis or \\
\hline
\end{tabular}

Box 4
Pneumonitis usually responds to prednisolone therapy, but fibrosis is unresponsive. Careful planning techniques should minimise this problem.

The addition of endobronchial radiotherapy following radical external beam therapy has yet to make a significant impact on survival rates, but further technical developments and greater experience in the integration of external beam therapy and endobronchial therapy may offer prospects of improved results. ${ }^{6}$

\section{PALLIATIVE RADIOTHERAPY}

\section{Palliation of the primary tumour}

Radiotherapy will alleviate many of the symptoms due to the primary tumour, particularly haemoptysis, major airway obstruction causing dyspnoea, cough, and pain (eg, due to direct invasion of the chest wall or other structures by the tumour). Superior mediastinal obstruction, which may be the presenting symptom of lung cancer, is also relieved by radiotherapy in $70-75 \%$ of cases. Adjunctive steroid therapy is often given during the course of radiotherapy for superior mediastinal obstruction.

Radiotherapy does not relieve symptoms such as recurrent laryngeal nerve palsy, diaphragmatic palsy or systemic symptoms such as depression and lethargy. Neuroendocrine syndromes and peripheral neuropathy are usually not alleviated by radiotherapy, but pain due to hypertrophic pulmonary osteoarthropathy can improve after a course of radiation to the primary tumour. ${ }^{18}$

Palliative radiotherapy treatments are generally less complex than for radical treatment, but careful planning is still required. The volume treated is determined using posterioanterio and lateral chest $\mathrm{X}$-rays as well as information obtained at bronchoscopy on the site and extent of disease, and from thoracic CT scans if available. The treatment volume usually encompasses the primary tumour and adjacent mediastinum. Relatively simple techniques are used, often anterior and posterior opposed beams. The doses given are much smaller than in radical treatment (eg, $17 \mathrm{~Gy}$ in two fractions given one week apart, ${ }^{9} 20 \mathrm{~Gy}$ in five fractions treating daily, or $30 \mathrm{~Gy}$ in 10 fractions, treating daily) and are given in a short overall treatment time. The intent is to cause limited tumour regression in order to abolish distressing symptoms. The tumour will usually regrow at an interval of time between three and 12 months following the radiotherapy.

Acute side effects are not marked (usually transient dysphagia and minimal fatigue), allowing most patients to be treated without requiring admission to hospital. Late effects including pulmonary fibrosis and, rarely, spinal cord myelitis.

In some patients, who have recurrent symptoms due to tumour regrowth, retreatment can be given. This may take the form of further external beam therapy but endobronchial radiotherapy can also effectively palliate symptoms such as haemoptysis, dyspnoea due to major airway obstruction, and cough in $60-80 \%$ of patients. ${ }^{10-13}$

\section{Palliation of metastatic symptoms}

Bone metastases Radiotherapy can effectively palliate painful bony metastases in up to $80 \%$ of patients, ${ }^{14}$ often using a single treatment of $8-10 \mathrm{~Gy}$ to the affected site.

Cerebral metastases The diagnosis usually follows CT scan of the brain in a lung cancer patient with neurological symptoms (eg, headaches, fits, focal neurological deficit). Initial management is with steroid therapy (eg, dexamethasone $4 \mathrm{mg}$ qds), but cranial irradiation is an effective method of reducing steroid dependence. Radiotherapy is usually given to the whole brain using laterally opposed fields, and moderate doses (eg, $20 \mathrm{~Gy}$ in five fractions given daily) are used. Patients who present with a solitary metastasis which is inoperable can be treated by new focal methods of radiotherapy, such as CT-guided stereotactic techniques, which provides a better dose distribution to the tumour and a reduced dose to the surrounding normal brain. ${ }^{15}$ Hair loss is a temporary though inevitable side effects of cranial irradiation but emesis and nausea are not often encountered.

Nodal and subcutaneous metastases These sites of metastases are not infrequent, and can be treated by radiotherapy, which will reduce pain and prevent fungation through the skin. 


\section{Case history 2 \\ - age: 73 years \\ - past medical history: bronchiectasis; right lower lobectomy 1950 \\ - February 1992: recurrent haemoptysis; chest X-ray normal \\ - October 1992: chest X-ray: right paratracheal mass; bronchoscopy:tumour right upper lobe orifice and right main bronchus; histology: adenocarcinoma; CT scan: invasion of mediastinum; T4 NO MO \\ - November 1992: palliative radiotherapy to right upper lobe and adjacent mediastinum, $30 \mathrm{~Gy}$ in 10 fractions; initial regression of tumour and cessation of haemoptysis \\ - October 1993: CT scan: mass right mid zone; bronchoscopy: tumour right bronchus intermedius \\ - November 1993: retreatment external beam radiotherapy; $24.25 \mathrm{~Gy}$ in 7 fractions; relief of dyspnoea \\ - October 1994: further haemoptysis; bronchoscopy: tumour at right upper lobe orifice; retreatment endobronchial brachytherapy; $15 \mathrm{~Gy}$ in one fraction at $1 \mathrm{~cm}$ from the endobronchial catheter}

\section{Box 5}

\begin{tabular}{|l|}
\hline Indications for palliation of \\
secondary tumours \\
\hline - painful bony metastases \\
- cerebral metastases \\
- nodal and subcutaneous metastases \\
\hline
\end{tabular}

Box 6

\begin{tabular}{|l|}
\hline Indications for supportive \\
care \\
\hline - symptomatic pleural effusion \\
- vocal cord palsy \\
- depression, anorexia, weight loss \\
- pain due to local infiltration \\
hypercalcaemia \\
\hline
\end{tabular}

Box 7

\begin{tabular}{|l|}
\hline $\begin{array}{l}\text { Management of } \\
\text { hypercalcaemia }\end{array}$ \\
\hline mild (serum calcium \\
$2.3-2.6$ mmol $^{-1}$ ): nil if \\
asymptomatic \\
- moderate $($ calcium \\
$2.7-3.0$ mmol $1^{-1}$ ): intravenous \\
fluids and steroids \\
- severe (calcium $>3$ mmol. $1^{-1}$ ): iv \\
fluids, steroids, bisphosphonates \\
\hline
\end{tabular}

Box 8

\section{Cytotoxic chemotherapy}

The use of cytotoxic chemotherapy has not resulted in improved survival of patients with advanced or metastatic non-small cell lung cancer ${ }^{16,17}$ Patients with cerebral or bony metastases are not curable even with systemic agents. Suitable palliation should be employed in these patients.

Many agents have been used in non-small cell lung cancer in the last two decades, both as single agents and in combinations. Most of these agents are associated with poor response rates $(<20 \%$ ), although there may be good individual responses. Five drugs have been identified as having response rates $>15 \%$ when used as single agents, ${ }^{18}$ namely, cisplatinum, ifosfamide, mitomycin, vinblastine, and vindesine. Of these cisplatinum has the highest response rate, of the order of $20-30 \%$, and this drug is often included in multi-agent regimens. Multi-agent regimens are reported as having higher response rates (up to $40 \%$ in some instances), but these have occurred in highly selected populations of patients. ${ }^{19,20}$ Toxicity from cytotoxic drugs may be considerable, especially in elderly patients, and thus they may not be appropriate for the majority of patients.

Novel cytotoxic agents are being developed constantly, and many Phase I and Phase II trials are in progress worldwide. It is hoped that developments in molecular biology will provide specific cytostatic therapies which will eventually improve the results obtained using conventional treatments.

\section{Surveillance}

Many patients with inoperable and incurable non-small cell lung cancer have few or no symptoms, and in some instances it may be appropriate not to give palliative treatment at presentation, but rather to wait until the patient has symptoms. There are few formal studies of surveillance, ${ }^{21}$ but there is little evidence that this approach is disadvantageous to the patients, providing that appropriate therapy is given without delay when symptoms do occur. The Medical Research Council are currently conducting a study of best supportive care against palliative treatment at presentation, and the results are awaited with interest.

\section{Supportive medical and surgical treatment}

Many patients will have symptoms requiring more general medical support. Symptomatic pleural effusions will require aspiration which may have to be repeated, and pleurodesis can be performed to prevent recurrent effusions. Dysphonia due to recurrent laryngeal nerve palsy may be improved after Teflon injection of the affected vocal cord. Depression and anorexia are common systemic symptoms which may be relieved by the judicious use of antidepressants (eg, prothiaden $75 \mathrm{mg}$ nocte) and prednisolone (10-15 mg daily), respectively.

Local pain caused by nerve infiltration (eg, from a Pancoast tumour infiltrating the brachial plexus) may be relieved by opioid analgesia and carbamazepine whilst awaiting a response to radiotherapy. Recurrent pain following radiotherapy can be relieved by appropriate nerve root blocks if opiates are not effective. Internal fixation of severe destructive bone metastases or pathological fractures due to bone metastases (eg, of the femur or hip) may be performed prior to radiotherapy to the affected site.

Hypercalcaemia is a common feature of non-small cell lung cancer which may require in-patient treatment with intravenous fluid replacement, diuretics, and steroids. If the hypercalcaemia is symptomatic (usually when the serum calcium level exceeds $3 \mathrm{mmol}^{-1}$ ) additional specific treatment using intravenous bisphosphonates (eg, disodium pamidronate $15-60 \mathrm{mg}$ over $12-24 \mathrm{~h}$ ) will be required to correct the biochemical abnormalities. ${ }^{22}$ The hypercalcaemia commonly recurs and in some instances it may be appropriate not to give specific treatment, but to continue supportive care as this is often a terminal event.

\section{New developments}

There are many new developments in the management of patients with inoperable non-small cell lung cancer, including improved imaging for staging and for radiotherapy planning, novel cytotoxic chemotherapeutic agents, and focal methods of increasing the dose of radiation given to local tumours without compromising normal tissue tolerance.

Different fractionation schemes for radical radiotherapy are being developed. These include shortened overall treatment time, often combined with multiple 


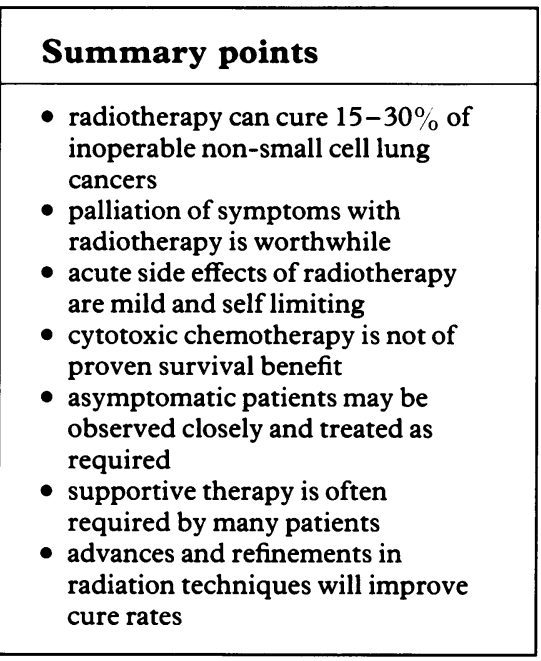

Box 9

1 Hoffmann TH, Randsell HT. Comparison of lobectomy and wedge resection for carcinoma of the lung. F Thorac Cardiovasc Surg 1980; 79: 211-7.

2 Armstrong JG, Minsky BD. Primary radiation therapy for stage I and II medically inoperable non small cell lung cancer. Cancer Treat Rev 1989; 6: 247-55.

3 Payne DG. Non small cell lung cancer: should unresectable stage III patients routinely receive high dose radiation therapy? $f$ Clin Oncol 1988; 6: $552-8$.

4 Saunders MI, Bennett MH, Dische S, et al. Primary tumour control after radiotherapy for carcinoma of the bronchus. Int $\mathcal{F}$ Radiat Oncol Phys Biol 1984; 10: 499-501.

5 Perez CA, Stanley K, Rubin P, et al. The Radiotherapy Oncology Group: patterns of umour recurrence after definitive irradiation for inoperable non-oat cell carcinoma of the ung. Int $\mathcal{f}$ Radiat Oncol Biol Phys 1980; 6: 987-94.

6 Speiser B, Spratling L. Remote afterloading brachytherapy for the local control of endobronchial carcinoma. Int $\mathcal{f}$ Radiat Oncol Biol Phys 1993; 25: 579-87.

7 Perez CA, Presant CA, van Amburg AL. Management of superior venal cava syndrome. Semin Oncol 1978; 5: 123-4.

8 Ginsberg RJ, Kris MG, Armstrong JG. Cancer of the lung. In: DeVita VT, Hellman $S$, Rosenberg SA (eds) Cancer: principles and practice of oncology. Philadelphia: JB Lipincott Co, tice of oncology.

daily fractions. ${ }^{23}$ This approach has been developed in response to the short tumour clonogen doubling times of non-small cell lung cancer which suggested that long treatment times were disadvantageous, allowing significant tumour cell division to continue during radiotherapy, and between cycles of chemotherapy.

9 Bleehan N. Inoperable non small cell lung cancer (NSCLC): a Medical Research Council randomised trial of palliative radiotherapy with 2 fractions or 10 fractions. $B r f$ Cancer $1991 ; 63$ : 265-70.

10 Burt P, O'Driscoll R, Notley M, Barber P, Stout $R$. Intraluminal irradiation for the palliation of lung cancer with the high dose rate microSelectlung cancer with the high dose

11 Hetzel MR, Smith SGT. Endoscopic palliation of tracheobronchial malignancies. Thorax 1991; 46: 325-33.

12 Mehta $M$, Shahabi S, Jarjour N, Kinsella $T$. Endobronchial irradiation for malignant airway obstruction. Int $\mathcal{F}$ Radiat Oncol Biol Phys 1989; 17: 847-51.

13 Speiser B. High dose rate brachytherapy of lung cancer: palliation or cure? Int $\mathcal{F}$ Radiat Onco Biol Phys 1994; 28: 781-2.

14 Price P, Hoskin PJ, Easton D, et al. Prospective trial of single and multifraction radiotherapy schedules in the treatment of painful bony metastases. Radiother Oncol 1986; 6: 247-55.

15 Flickinger JC, Kondziolka D, Lunsford LD, al. A multiinstitutional experience al. A al. A solitary brain 28: 797-802. 28: 797-802.

16 Cellerino R, Tummarello D, Piga A. Chemotherapy or not in advanced non small cel lung cancer? Lung Cancer 1990; 6: 99-109.
17 Grilli R, Oxman AD, Julian JA. Chemotherapy for advanced non small cell lung cancer. How much benefit is enough? 7 Clin Oncol 1993; 11: 1866-72.

18 Kris M, Cohen E, Gralla R. An analysis of 134 phase II trials in non small cell lung cancer. IVth World Conference on Lung Cancer Toronto 1985, 39.

19 Donnalieu N, Paesman SM, Saulier J. Chemotherapy of non small cell lung cancer according to disease extent. A meta-analysis of the literature. Lung Cancer 1991; 7: 243-52.

20 Arriagada R, Le Chevalier T, Quoix E, et al. ASTRO plenary: effective chemotherapy on locally advanced non small cell lung cancer. A randomised study of 353 patients. Int $\mathcal{F}$ Radiat Oncol Phys Biol 1991; 20: 1183-90.

21 Carroll M, Morgan SA, Yarnold JR et al. Prospective evaluation of a watch policy in patients with inoperable non small cell lung cancer. Eur $f$ Cancer Clin Oncol 1986; 22: 1353-6.

22 Nussbaum SR, Mallette L, Gagel R, et al. Single dose treatment of hypercalcaemia of malignancy with aminohydroxyproplidene bisphosphonate with aminohydroxyproplidene bisphosph

23 Saunders MI, Dische S, Grosche E, et al. Experience with CHART. Int $\mathcal{f}$ Radiat Oncol Phys Biol 1991; 21: 871-8. 